Supporting Information

\title{
Directed evolution and rational design of mechanosensitive channel MscCG2 for improved glutamate excretion efficiency
}

Zhihua Nie ${ }^{1,2, \#,}$ Pi Liu' ${ }^{1,2, \#,}$ Yu Wang ${ }^{1,2}$, Xuan Guo ${ }^{1,2}$, Zijian Tan ${ }^{1,2}$, Jie Shen ${ }^{1,2}$, Zijing

Tang $^{1,2}$, Jianping Lin ${ }^{1,2}$, Jibin Sun ${ }^{1,2}$, Ping Zheng ${ }^{1,2, *}$, Leilei Zhu ${ }^{1,2 *}$

${ }^{1}$ Tianjin Institute of Industrial Biotechnology, Chinese Academy of Sciences, Tianjin 300308, China.

${ }^{2}$ National Technology Innovation Center of Synthetic Biology, Tianjin 300308, China.

\# These authors contributed equally.

*Corresponding author:

Prof. Dr. Ping Zheng

Tianjin Institute of Industrial Biotechnology, Chinese Academy of Sciences, Tianjin 300308, China

Email: zheng_p@tib.cas.cn

Tel: $+86-2224821994$

Prof. Dr. Leilei Zhu

Tianjin Institute of Industrial Biotechnology, Chinese Academy of Sciences, Tianjin 300308, China

E-mail: zhu_11@tib.cas.cn

Tel: $+86-2224828796$

Number of pages: 5

Number of figures: 1

Number of tables: 2 


\section{Contents}

Figure S1. Sequence and secondary structure alignment of sequence of MscCG2, ITASSAR predicted structure model of MscCG2, homology template of PDB 6RLD and PDB 3T9N

Figure S2. Structure check of homology model of MscCG2

Figure S3. Glutamate export rate of MscCG2 WT, variant M1, M2 and M3

Figure S4. Excretion of aspartate by MscCG2 WT, variants M1, M2 and M3

Figure S5. The cell density during the growth and glucose consumption of MscCG2 WT, variants M1, M2, M3 and A151V in shake flask

Figure S6. The cell density during the growth of combinations L165P/I269V, L165P/F279L, I269V/F279L and L165P/I269V/F279L in shake flask

Figure S7. The effect of substituted Ala and Val for Phe149 on the glutamate excretion

Figure S8. The cell density during the growth of variants M4 (F149L), combinations

L165P/F149L, I269V/F149L and F279L/F149L in shake flask

Table S1. PCR Primers used in this study

Table S2. Bacterial strains and plasmids used in this study

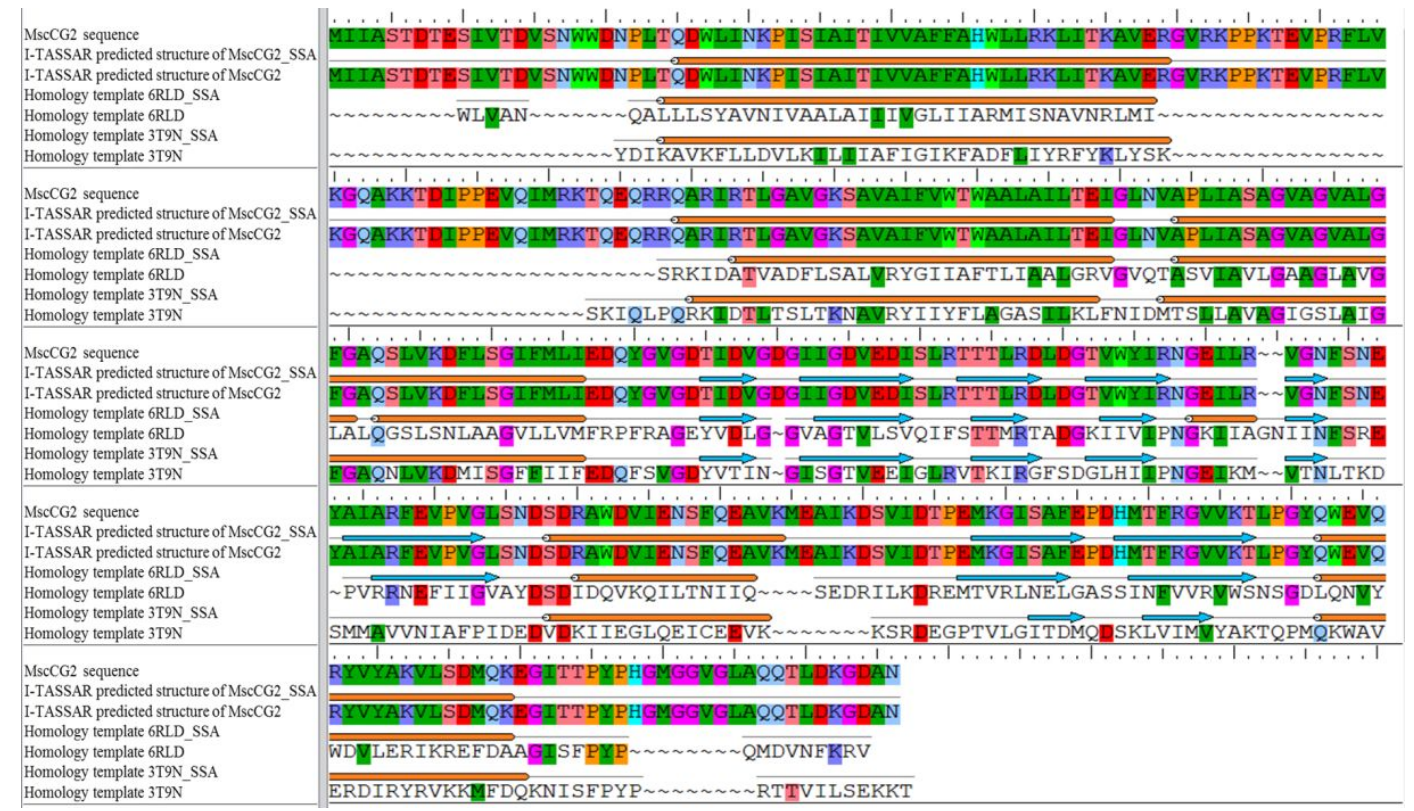

Figure S1. Sequence and secondary structure alignment of sequence of MscCG2, I-

TASSAR predicted structure model of MscCG2, homology template of PDB 6RLD 
and PDB 3T9N.

Program: ERRAT2

File: $\mathrm{msccg} 2$ _check

Chain\#:

Overall quality factor ${ }^{\star \star}: 94.628$

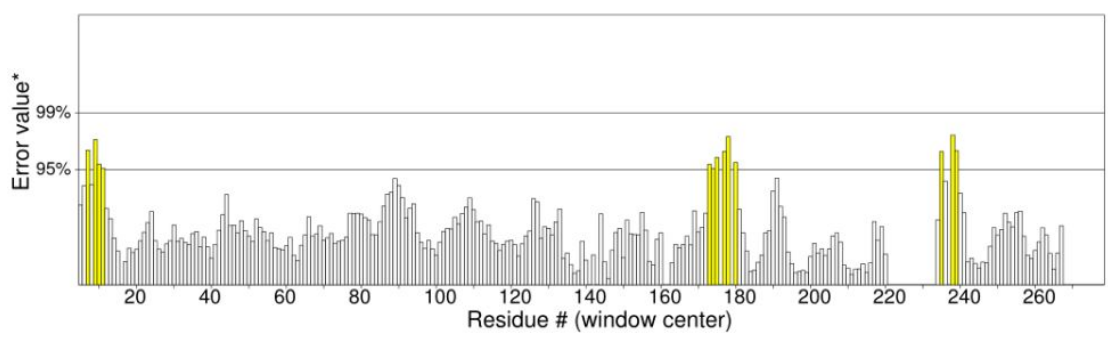

"On the error axis, two lines are drawn to indicate the confidence with
which it is possible to reject regions that exceed that error value.

"Expressed as the percentage of the protein for which the calculated

"Expressed as the percentage of the protein for which the calculated
error value falls below the $95 \%$ rejection limit. Good high resolution
structures generally produce values around $95 \%$ or higher. For low

structures generally produce values around $95 \%$ or higher. For lower

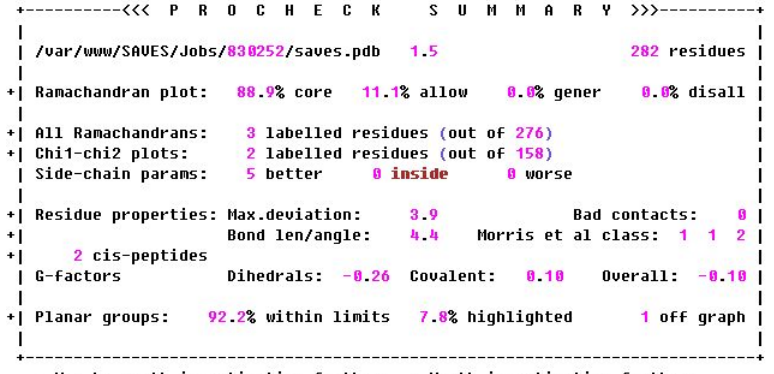

+ May be worth inuestigating further. * Worth inuestigating further.

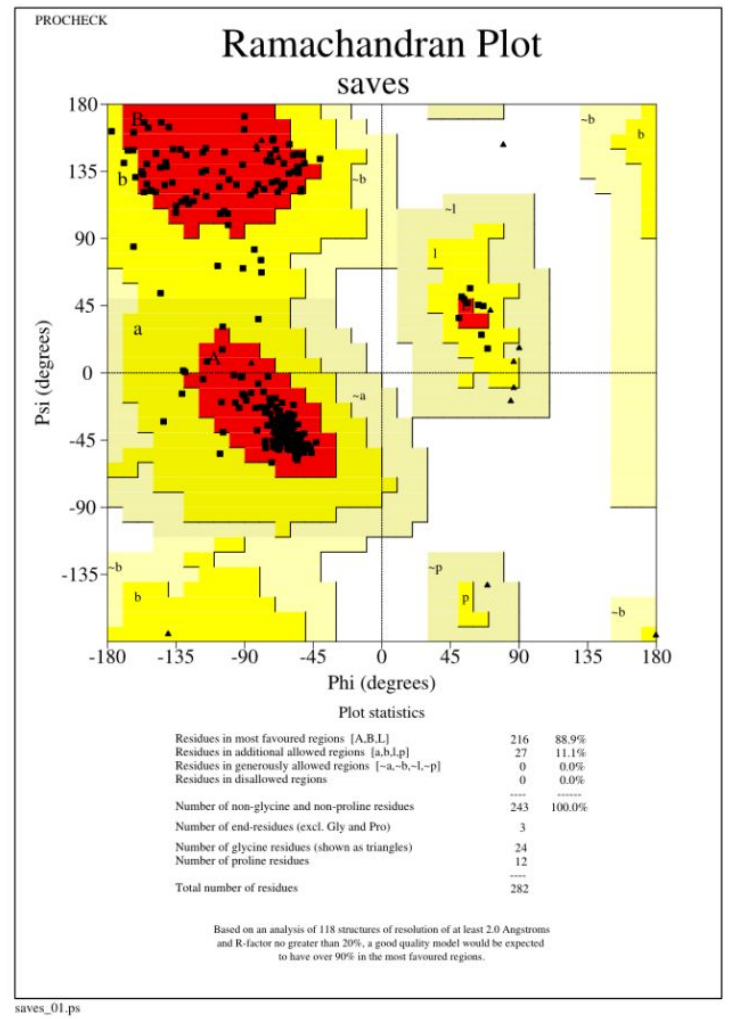

Figure S2. Structure check of homology model of MscCG2. 


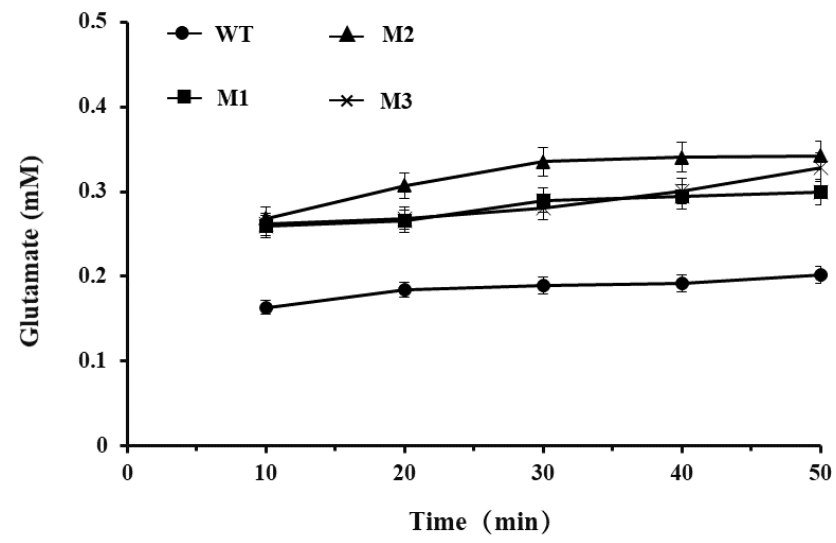

Figure S3. Glutamate export rate of MscCG2 WT, variant M1, M2 and M3.

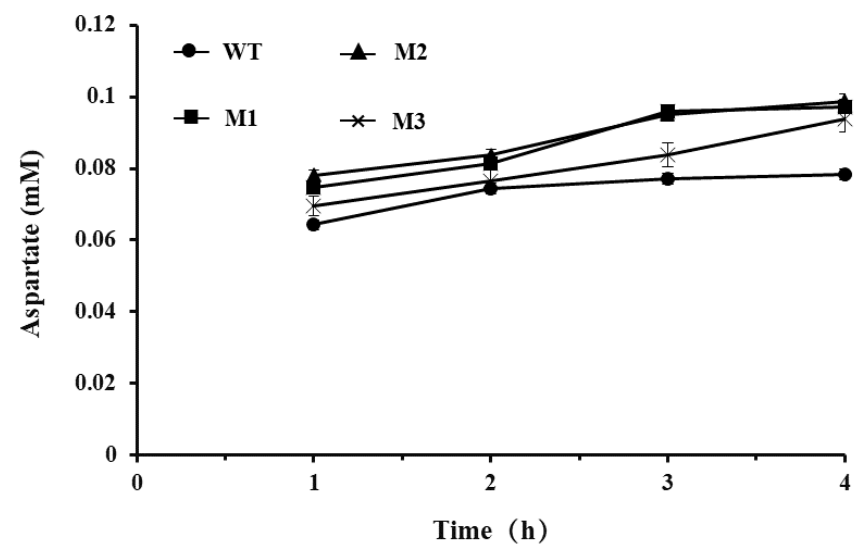

Figure S4. Excretion of aspartate by MscCG2 WT, variants M1, M2 and M3.

A

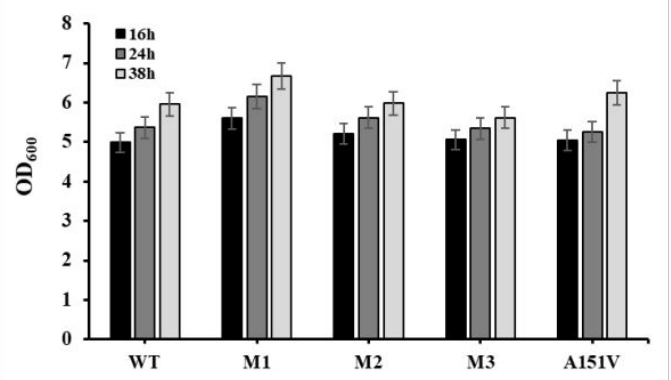

B

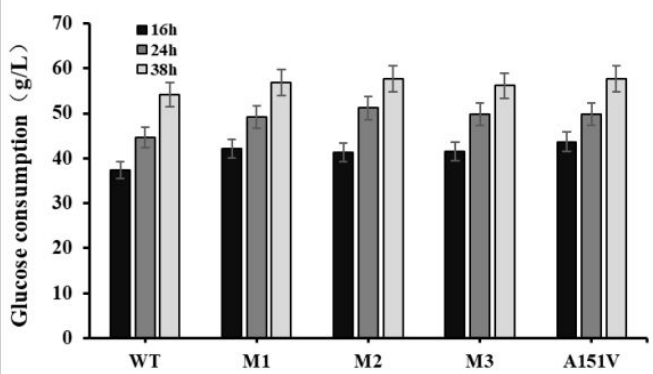

Figure S5. The cell density during the growth and glucose consumption of MscCG2 WT, variants M1, M2, M3 and A151V in shake flask. 
A

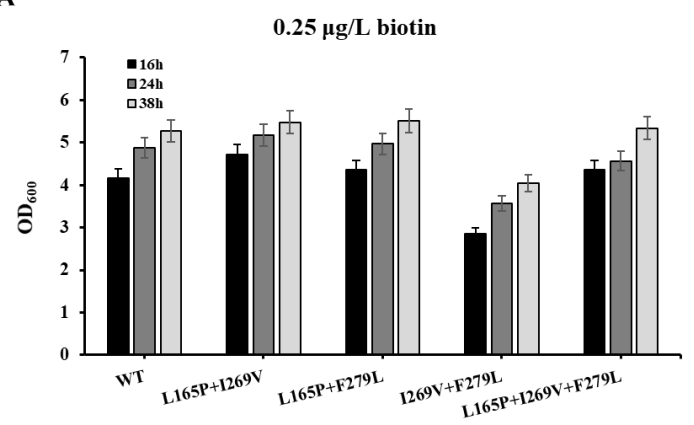

B

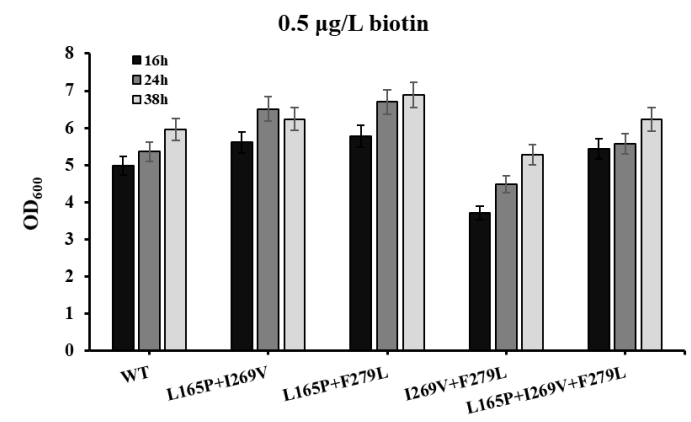

Figure S6. The cell density during the growth of combinations L165P/I269V, L165P/F279L, I269V/F279L and L165P/I269V/F279L in shake flask.

A

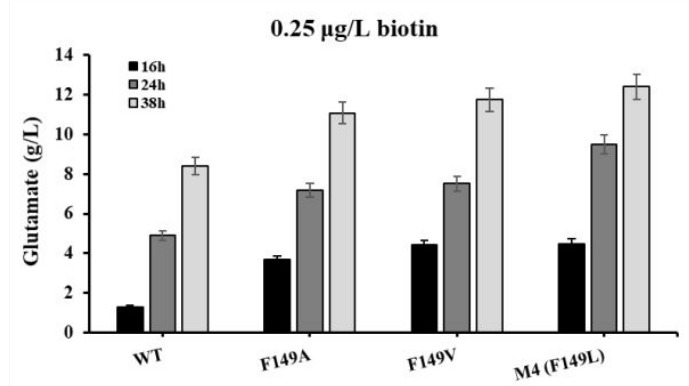

B

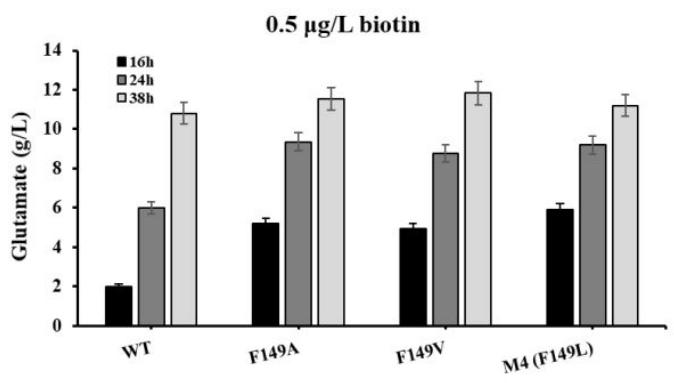

Figure S7. The effect of substituted Ala and Val for Phe149 on the glutamate excretion.

A

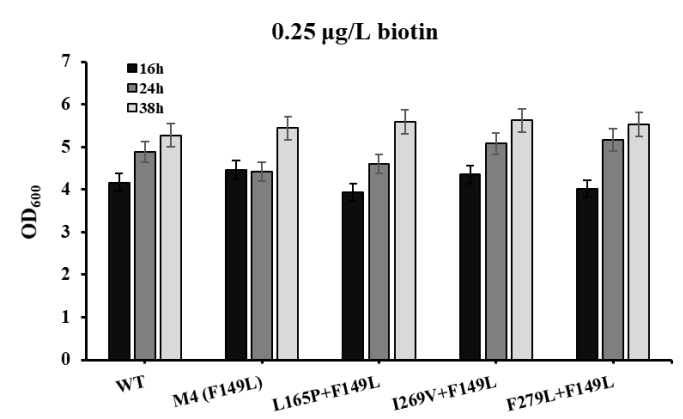

B

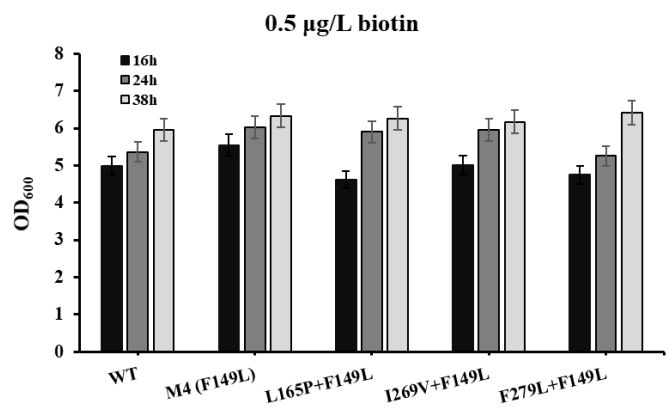

Figure S8. The cell density during the growth of variants M4 (F149L), combinations L165P/F149L, I269V/F149L and F279L/F149L in shake flask. 
Table S1. PCR Primers used in this study

\begin{tabular}{|c|c|c|}
\hline Relevance & Primer & Sequence (5'-3') \\
\hline$m s c C G 2$ amplification and & $m s c C G 2-\mathrm{F}$ & CAGACCATGGAATTCGAGCTCATGATTATCGCTAGTACAGA \\
\hline \multicolumn{3}{|l|}{ epPCR } \\
\hline & $m s c C G 2-\mathrm{R}$ & AGGTCGACTCTAGAGGATCCTTAGTTGGCGTCGCCTTTAT \\
\hline \multirow[t]{2}{*}{ pTRCmob amplification } & pTRCmob-F & GATAAAGGCGACGCCAACTAAGGATCCTCTAGAGTCGACCT \\
\hline & pTRCmob-R & TCTGTACTAGCGATAATCATGAGCTCGAATTCCATGGTCTG \\
\hline pTRCmob-mscCG $2^{\mathrm{A} 151 \mathrm{~V}}$ & $m s c C G 2^{\mathrm{A}^{151 \mathrm{v}_{-}} \mathrm{F}}$ & GCGCTTGGTTTTGGCGTACAGTCGCTGGTGAAG \\
\hline \multicolumn{3}{|l|}{ construction } \\
\hline & $m s c C G 2^{\mathrm{A} 151 \mathrm{~V}_{-} \mathrm{R}}$ & CTTCACCAGCGACTGTACGCCAAAACCAAGCGC \\
\hline pTRCmob- & $m s c C G 2^{\mathrm{L} 165}-\mathrm{F}$ & CAGCGGTATTTTTATGCCCATTGAGGATCAATACGG \\
\hline \multicolumn{3}{|l|}{$m s c C G 2^{\mathrm{L} 165 \mathrm{P}+1269 \mathrm{~V}}$ construction } \\
\hline & $m s c C G 2^{\mathrm{L} 165 \mathrm{P}}-\mathrm{R}$ & CCGTATTGATCCTCAATGGGCATAAAAATACCGCTG \\
\hline pTRCmob- & $m s c C G 2^{\mathrm{L} 165 \mathrm{P}-\mathrm{F}}$ & CAGCGGTATTTTTATGCCCATTGAGGATCAATACGG \\
\hline \multicolumn{3}{|l|}{$m s c C G 2^{\mathrm{L} 165 \mathrm{P}+\mathrm{F} 279 \mathrm{~L}}$ construction } \\
\hline & $m s c C G 2^{\mathrm{L} 165 \mathrm{P}}-\mathrm{R}$ & CCGTATTGATCCTCAATGGGCATAAAAATACCGCTG \\
\hline pTRCmob- & $m s c C G 2^{1269 \mathrm{~V}_{-} \mathrm{F}}$ & CCTGAGATGAAGGGCGTCTCTGCATTCGAACCT \\
\hline \multicolumn{3}{|l|}{$m s c C G 2^{\mathrm{L} 165 \mathrm{P}+\mathrm{I} 269 \mathrm{~V}+\mathrm{F} 279 \mathrm{~L}}$} \\
\hline \multicolumn{3}{|l|}{ construction } \\
\hline & $m s c C G 2^{1269 \mathrm{v}_{-} \mathrm{R}}$ & AGGTTCGAATGCAGAGACGCCCTTCATCTCAGG \\
\hline pTRCmob-mscCG $2^{\mathrm{F} 149 \mathrm{~L}}$ & $m s c C G 2^{\mathrm{F} 149 \mathrm{~L}-\mathrm{F}}$ & GGTGTTGCGCTTGGTCTTGGCGCACAGTCGCTG \\
\hline \multicolumn{3}{|l|}{ construction } \\
\hline & $m s c C G 2^{\mathrm{F} 149 \mathrm{~L}-\mathrm{R}}$ & CAGCGACTGTGCGCCAAGACCAAGCGCAACACC \\
\hline pTRCmob- & $m s c C G 2^{\mathrm{F} 149 \mathrm{~L}_{-} \mathrm{F}}$ & GGTGTTGCGCTTGGTCTTGGCGCACAGTCGCTG \\
\hline \multicolumn{3}{|l|}{$m s c C G 2^{\mathrm{L} 165 \mathrm{P}+\mathrm{F} 149 \mathrm{~L}}$ construction } \\
\hline & $m s c C G 2^{\mathrm{F} 149 \mathrm{~L}-\mathrm{R}}$ & CAGCGACTGTGCGCCAAGACCAAGCGCAACACC \\
\hline pTRCmob- & $m s c C G 2^{\mathrm{F} 149 \mathrm{~L}_{-} \mathrm{F}}$ & GGTGTTGCGCTTGGTCTTGGCGCACAGTCGCTG \\
\hline \multicolumn{3}{|l|}{$m s c C G 2^{1269 \mathrm{~V}+\mathrm{F} 149 \mathrm{~L}}$ construction } \\
\hline & $m s c C G 2^{\mathrm{F} 149 \mathrm{~L}-\mathrm{R}}$ & CAGCGACTGTGCGCCAAGACCAAGCGCAACACC \\
\hline
\end{tabular}




\begin{tabular}{|c|c|c|}
\hline pTRCmob- & $m s c C G 2^{\mathrm{F} 149 \mathrm{~L}_{-} \mathrm{F}}$ & GGTGTTGCGCTTGGTCTTGGCGCACAGTCGCTG \\
\hline \multicolumn{3}{|c|}{$m s c C G 2^{\mathrm{F} 279 \mathrm{~L}+\mathrm{F} 149 \mathrm{~L}}$ construction } \\
\hline & $m s c C G 2^{\mathrm{F} 149 \mathrm{~L}-\mathrm{R}}$ & CAGCGACTGTGCGCCAAGACCAAGCGCAACACC \\
\hline
\end{tabular}

Table S2. Bacterial strains and plasmids used in this study

\begin{tabular}{lll}
\hline Strain or plasmid & Relevant characteristic & Reference or source \\
\hline E.coli BL 21-Gold (DE3) & General cloning host & Lab collection \\
\hline
\end{tabular}


C. glutamicum

$\mathrm{ATCC} 13032 \Delta m s c C G$

pTRCmob

pTRCmob-mscCG2

pTRCmob- $m s c C G 2^{\mathrm{M} 1}$

pTRCmob- $m s c C G 2^{\mathrm{M} 2}$

pTRCmob-mscCG2 $2^{\mathrm{M} 3}$

pTRCmob-mscCG2 $2^{\mathrm{A} 151 \mathrm{~V}}$

pTRCmob- $m s c C G 2^{\mathrm{L} 165 \mathrm{P}+\mathrm{I} 269 \mathrm{~V}}$

pTRCmob- $m s c C G 2^{\mathrm{L} 165 \mathrm{P}+\mathrm{F} 279 \mathrm{~L}}$

pTRCmob- $m s c C G 2^{1269 \mathrm{~V}+\mathrm{F} 279 \mathrm{~L}}$

pTRCmob-

$m s c C G 2^{\mathrm{L} 165 \mathrm{P}+1269 \mathrm{~V}+\mathrm{F} 279 \mathrm{~L}}$

pTRCmob-mscCG2 ${ }^{\text {F149L (M4) }}$

pTRCmob- $m s c C G 2^{\mathrm{L} 165 \mathrm{P}+\mathrm{F} 149 \mathrm{~L}}$

pTRCmob-mscCG $2^{\mathrm{I} 269 \mathrm{~V}+\mathrm{F} 149 \mathrm{~L}}$

pTRCmob-mscCG $2^{\mathrm{F} 279 \mathrm{~L}+\mathrm{F} 149 \mathrm{~L}}$
ATCC13032 with its $m s c C G$ deleted

Lab collection

Expression vector

Lab collection

pTRCmob derivative, carries $m s c C G 2$

this study

pTRCmob derivative, carries $m s c C G 2$ variant

this study

M1

pTRCmob derivative, carries $m s c C G 2$ variant this study

M2

pTRCmob derivative, carries $m s c C G 2$ variant this study

M3

pTRCmob derivative, carries $m s c C G 2$ variant this study

pTRCmob derivative, carries $m s c C G 2$ variant this study

pTRCmob derivative, carries $m s c C G 2$ variant this study

pTRCmob derivative, carries $m s c C G 2$ variant this study

pTRCmob derivative, carries $m s c C G 2$ variant this study

pTRCmob derivative, carries $m s c C G 2$ variant this study

pTRCmob derivative, carries $m s c C G 2$ variant this study

pTRCmob derivative, carries $m s c C G 2$ variant this study

pTRCmob derivative, carries $m s c C G 2$ variant this study 